\section{Spectral Response of Lead Selenide}

MrCRo-CRYSTALLINE layers of lead selenide are known to show photo-conductivity when illuminated by infra-red radiation, in a manner similar to that shown by lead sulphide. Measurements made in Germany during the War indicate that for lead selenide the long wave-length limit of sensitivity lies farther towards the far infra-red ${ }^{1}$. More recent experiments by Blackwell, Simpson and Sutherland 2 on the spectral response of lead selenide confirm this. Up to the present, however, the accurate determina. tion of the long-wave limit has been impossible because of the very strong absorption in this region of the spectrum of 'Pyrex' and other glasses from which the photo-conductive cells have been made. We have recently developed a technique whereby a sensitive layer may be made in a cell with a window of KRS5 material (a mixture of thallium bromide and iodide which has a uniform transmission of about 65 per cent from 1 to $35 \mu$ ).

The cell is made in an envelope of the usual Dewar flask type except that the outer wall is formed into an elongated neck opposite the layer electrodes, which are on the inner wall. Initially a layer of lead selenide is evaporated on to the electrodes in vacuo, and then the end of the glass neck is cut off, ground flat, and the window sealed on with vacuum wax. The layer is then treated by heating to a temperature just below its subliming point (about $405^{\circ} \mathrm{C}$.) in vacuo for several hours. For this treatment the inside of the Dewar vessel is filled with a molten (low melting point) alloy maintained at the appropriate temperature by means of a small immersion heater. Finally, the layer must be recrystallized by evaporating on to the outer wall of the flask and then back on to the electrodes, when a sensitive, highresistance layer is obtained. Throughout these processes the window is kept cool by standing in cold water.

The accompanying diagram shows the spectral response of lead selenide for constant incident energy at $195^{\circ} \mathrm{K}$. and $90^{\circ} \mathrm{K}$. The shift to longer wave-lengths on cooling is analogous to that observed for lead sulphide ${ }^{3}$. Taking the flat portion of the curve as the datum-level, the response at liquid oxygen tem-

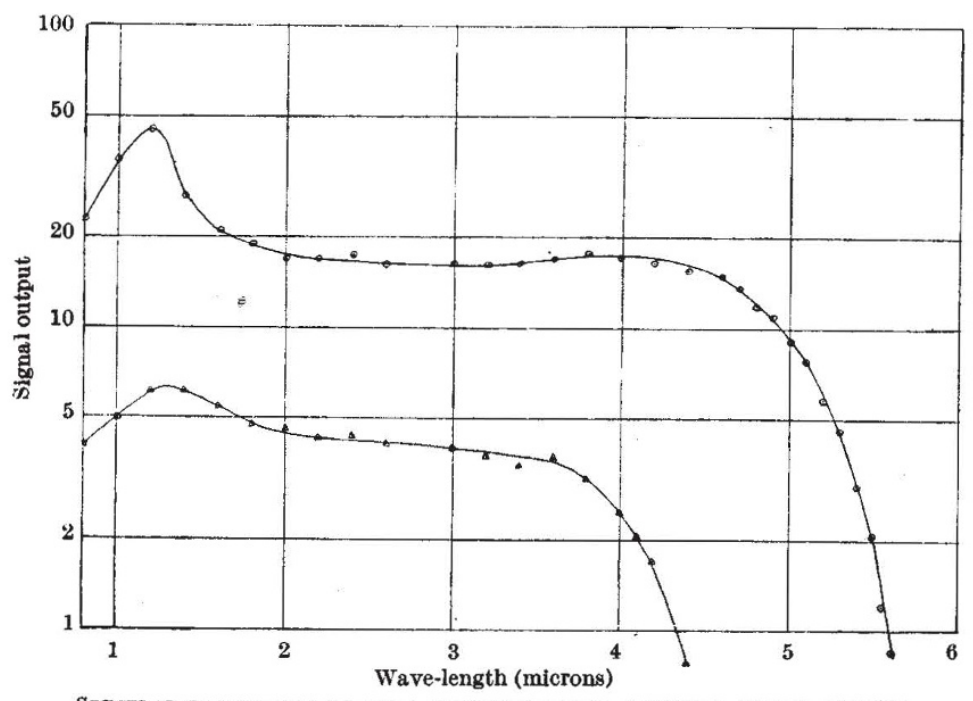
SPECTRAL SENSITIVITY OF LRAD SELENIDE LAYER, SHOWING SIGNAL OUTPUT
FROX THE LAYRR FOR CONSTANT INCIDENT RNERGY. $\odot, 90^{\circ} \mathrm{K}$.; $\Delta, 195^{\circ} \mathrm{K}$. (on same seale) perature is down to 50 per cent at $5 \cdot 0 \mu$, indicating about $1 \cdot 0 \mu$ shift from the $195^{\circ} \mathrm{K}$. curve.

The peak found at $1 \cdot 2 \mu$ is believed to be due to impurities and not inherently characteristic of the main lattice. At longer wave-lengths the response curve differs somewhat from that of other workers ${ }^{1,2}$, who find a slight secondary peak. The presence of this peak may perhaps be connected with the varying transmission of the layer with wave-length. If the layer is too thin, all the incident energy is no longer absorbed at the longer wave-lengths, giving a false impression of the shape of the spectral sensitivity curve.

The layer which is the subject of this note was therefore made relatively thick (estimated as somewhat greater than $1 \mu$ from the mass and area of the layer). Previous measurements in this laboratory have shown that layers appreciably thinner than this have considerable transmission in the region of $5 \mu$.

The spectrometer used was a double-dispersion instrument using lithium fluoride prisms and having a high degree of freedom from scatter.

Our thanks are due to Dr. G. B. B. M. Sutherland and Mr. O. Simpson, of the University of Cambridge, for communicating to us, before publication, their method of making sensitive lead selenide cells ${ }^{4}$ and their recent spectral response measurements ${ }^{2}$; also to the Ministry of Supply for permission to publish this letter.

\section{T. S. Moss}

R. P. Chasmar

Physics Department,

Telecommunications Research Establishment, Great Malvern.

Nov. 5.

'Elliot, "Electronics", chapter 3 (London : Pilot Press, 1947).

"Blackwell, Simpson and Sutherland, [Nature, 160, 793 (1947) ].

${ }^{3}$ Moss, Nature, 159, 476 (1947).

- Simpson, [Nature, 160, 791 (1947)].

\section{Pinhole Radiography of Magnetrons}

'Pinhole RADIOGRAPHY' is extensively used ${ }^{1}$ to study the size and shape of the focal spot of an X-ray tube. Where electrons strike the anode, X-rays are emitted in all directions; a camera, with a pinhole in a sheet opaque to $\mathrm{X}$-rays in place of the lens, can be used to record an X-ray image of the bombarded area.

Hitherto, magnetrons ${ }^{2}$ have operated at anode voltages too low for X-rays to escape from the tube envelope in appreciable intensity. We have recently been working on a high-power $(2,000 \mathrm{~kW}$.) pulsed $10 \mathrm{~cm}$. magnetron, operating at $47 \mathrm{kV}$. anode voltage, 90 -amp peak, 135-m.amp. mean anode current ${ }^{3}$. Having ob. served X-ray emission from this valve $\left(10^{-5} \mathrm{r}\right.$. $/$ sec. at 1 metre distance $)$ we decided to make the following experiment.

The normal electromagnet gap of $8 \mathrm{~cm}$. was increased to $11.5 \mathrm{~cm}$., while maintaining the magnetic field at 1,600 oersteds. The valve was set up with one end-plate against a pole-face. A sheet of lead, $2 \mathrm{~mm}$. thick, was placed against the other end-plate, and contained a pinhole situated on the axis of the valve. 